\title{
Faune aquatique et qualité de l'eau des puits et sources de la région d'Oum-El-Bouaghi (Nord-Est algérien)
}

\section{Aquatic fauna and water quality from wells and springs in the region of Oum-El-Bouaghi (North-East of Algeria)}

\author{
D. Merzoug( ${ }^{(1)}$, A. Khiari(1) A. Aït Boughrous(2), C. Boutin ${ }^{(3,4)}$ \\ (1) Laboratoire «Ressources naturelles et Aménagement des milieux sensibles », Université Larbi Ben M'hidi, \\ BP 358, Oum-El-Bouaghi, Algérie \\ merzmoi@yahoo.fr \\ (2) Université Cadi Ayyad, Faculté des Sciences Semlalia, Département de Biologie, Laboratoire d'Hydrobio- \\ logie, Écotoxicologie et Assainissement, BP 2390, Boulevard Prince Moulay Abdellah, Marrakech, Maroc \\ (3) Université Paul Sabatier, UMR CNRS-UPS-INP n 5245, Laboratoire d'Écologie Fonctionnelle (ÉCOLAB), \\ Équipe Stygobiologie, 39 allées Jules Guesde, 31400 Toulouse, France \\ (4) 48 rue Mouffetard, 75005 Paris, France \\ boutin@cict.fr
}

Résumé - Une étude récente réalisée dans la région d'Oum-El-Bouaghi, dans le Nord-Est de l'Algérie, avait comme objectif de rechercher et de préciser la relation pouvant exister entre la qualité de l'eau des puits et des sources et la diversité de la faune aquatique présente dans ces habitats. Pour cela une quinzaine de stations (16 puits et 2 sources) ont fait l'objet, périodiquement, d'une étude de la qualité de l'eau et d'un échantillonnage de la faune. Les stations retenues ont été choisies en raison de la diversité de leur faune mais aussi et surtout parce qu'elles présentaient entre elles certaines différences évidentes (profondeur de la nappe, nature du substratum, protection des puits et utilisation de l'eau par la population locale), qui pouvaient laisser espérer une certaine diversité. Les principales composantes physico-chimiques de la qualité de l'eau ont donc été mesurées puis une analyse en composantes principales (ACP) a été réalisée à partir des valeurs moyennes de chaque paramètre; on a pu révéler ainsi l'existence d'une variation spatiale relativement marquée de ces descripteurs, faisant apparaître quatre groupes de stations très inégaux et bien différenciés : un premier groupe de 11 puits situés à l'aval hydraulique de la zone d'étude, où l'eau présente des teneurs élevées en ions azotés et phosphorés indicateurs de pollution; à l'opposé un autre groupe de 5 puits, situés plus en amont, où l'eau présente au contraire de faibles concentrations de substances azotées et d'ions phosphorés, donc des stations peu ou pas polluées; enfin deux autres groupes bien séparés, chacun correspondant à une source, dont l'eau est aussi de qualité relativement bonne. La faune aquatique de ces puits et sources a également été échantillonnée de façon périodique. La richesse faunistique globale des stations apparaît faiblement corrélée avec la qualité de l'eau, mais en revanche la richesse spécifique de la faune stygobie (celle qui vit dans la nappe phréatique) et plus encore l'abondance de ces espèces stygobies, diminuent de 
façon significative avec la pollution. La faune stygobie dans son ensemble, et particulièrement le groupe des Crustacés péracarides, apparaît ainsi comme un très bon indicateur de la qualité de l'eau de ces puits et des sources.

Mots clés - biodiversité, puits, faune stygobie, espèces indicatrices, qualité des eaux souterraines

\begin{abstract}
A recent study, performed in the region of Oum-El-Bouaghi, North-Eastern Algeria, aimed at showing the possible relationships between the water quality of wells and springs and the diversity of the aquatic fauna which occurs in these habitats. To this end, the water quality and the fauna were regularly investigated in several stations (16 wells and 2 springs) selected in the region. The stations were chosen considering visible differences related to both their fauna and also some evident characteristics i.e. water table depth, nature of geological substratum, protection and human use, so that it was possible to expect certain diversity. The main physicochemical characteristics of water were measured and a Principal Component Analysis (PCA) was performed with the mean values of each variable. The PCA indicated an appreciable spatial variation of water quality related to the values of some hydrological variables and clearly showed four groups of stations: a first group of 11 wells located in the downstream part of the study area, where water exhibited a high level of nitrogenous and phosphoric ions revealing water pollution. In contrast, another group of 5 wells located more upstream and where the water, containing only very low concentrations of nitrogenous and phosphoric ions, is of good quality. Finally two other groups are well separated and include only one spring each, where water is also of good quality. Likewise, the fauna of each station was regularly sampled. The total faunal richness of stations was poorly correlated with water quality but, in contrast, the specific richness of the stygobiontic fauna (the subterranean species living in groundwater), and moreover the abundance of these stygobiontic species decreased significantly in case of water pollution. Thus the stygobiontic fauna and especially the peracarid crustaceans appear to be good indicators of water quality in the studied wells and springs.
\end{abstract}

Key words - biodiversity, wells, stygobiontic fauna, indicator species, groundwater quality

\section{INTRODUCTION}

Les eaux souterraines représentent au total environ $97 \%$ du total des eaux douces continentales liquides (Bosca, 2002) et la présence de l'Homme ainsi que ses possibilités de survie dépendent un peu partout sur le globe de l'existence et de la qualité de cette ressource pourtant limitée et fragile (Boutin, 1987) puisque 75 à $90 \%$ de la population mondiale utilisent une eau d'origine souterraine. C'est dire l'importance de l'étude des nappes souterraines exploitables dans toutes leurs composantes, en vue d'une meilleure connaissance de ces écosystèmes et si possible d'introduire des concepts scientifiques nouveaux pouvant avoir leur utilité en matière d'aménagement, de surveillance, de gestion, de protection et de conservation de cette ressource (Danielopol et al., 2004).

Les recherches écologiques concernant la faune aquatique souterraine et plus particulièrement celle des nappes phréatiques accessibles au niveau des puits ordinaires et accessoirement des sources, représentent un des aspects de la phréatobiologie appliquée qui a connu depuis quelques dizaines d'années un développement 
important dans le monde entier. En fait les puits ordinaires, de même que les sources constituent des écotones (Vandel, 1964 ; Dalmas, 1972, 1973; Gibert et al., 1997) qui abritent un assemblage faunistique comportant à côté des espèces aquatiques d'origine superficielle, variables selon les lieux et suivant l'état de protection du site, des espèces stygobies présentes aux alentours dans la nappe phréatique. On s'est aperçu assez rapidement que ce sont précisément ces dernières qui semblent, plus que les espèces aquatiques épigées, en relation avec la qualité des eaux souterraines (Boutin, 1984). Pour ces raisons, on s'est intéressé de plus en plus à la faune des milieux aquatiques souterrains, notamment à celle des nappes phréatiques ainsi qu'à celle du sousécoulement des cours d'eau, en utilisant diverses méthodes proposées notamment par des chercheurs tels que Cvetkov (1968), Bou (1974), Boutin \& Boulanouar (1983). Ces recherches sur l'écologie des milieux aquatiques hypogés ont fait l'objet de plusieurs synthèses parmi lesquelles il faut citer celles de Delamare Deboutteville (1960), Coineau (1971), Botosanéanu (1971, 1986), Gibert (1986), Coineau \& Boutin (1992), Gibert et al. (1994), Juberthie \& Decu (1994, 1998, 2001), Wilkens et al. (2000), Gunn (2004), Culver \& White (2005).

Les recherches stygobiologiques régionales - incluant l'étude faunistique des puits - ont connu un essor important sur les divers continents. Ainsi, en Amérique de nombreuses études ont été entreprises, notamment par Bousfield (1958), Maguire (1964), Holsinger (1972, 1986, 1994, 2000), Culver (1982), Koenemann \&
Holsinger (2001), et en Afrique subsaharienne par Magniez (1979) et récemment par Zébazé-Togouet et al. (2009).

$\mathrm{Au}$ Maghreb on peut citer les travaux déjà anciens de Racovitza (1912) et de Monod (1924, 1927) ainsi que les prospections plus récentes de Pesce et al. (1981) et, en particulier au Maroc, les travaux de Nourrisson (1956), Karaman \& Pesce (1980), Boutin \& Boulanouar (1983), Boulanouar (1986 et 1995), Messouli (1984, 1988, 1994), Boutin et Messouli (1988a, 1988b), Boulal (1988, 2002), Boutin \& Idbennacer (1989), Idbennacer (1990), Boutin (1993), Fakher El Abiari (1999), Aït Boughrous et al. (2007).

En Algérie on ne dispose à ce jour que de très peu de données sur la faune stygobie car seules des investigations ponctuelles et souvent anciennes ont été réalisées par divers chercheurs de passage, européens en général : Racovitza (1912), Monod (1930), Gauthier (1928), Nourrisson (1956), Delamare Deboutteville (1960), puis Pesce et al. (1981) et Dumont (1981). Cependant des travaux ont été entrepris récemment en Algérie par l'équipe de l'Université de Tlemcen (Belaidi-Aliane, 2004) et l'on doit aussi signaler la synthèse sur les faunes souterraines d'Algérie, publiée par Decu et al. (2001).

Enfin la faune aquatique des puits et des sources de la région d'OumEl-Bouaghi, dans le Nord-Est algérien, région encore inexplorée à ce jour, vient de faire l'objet d'une série d'investigations dont on trouvera ici les premiers résultats. II nous a semblé utile en effet d'envisager dans cette région une étude faunistique des puits et des sources, ainsi qu'une 


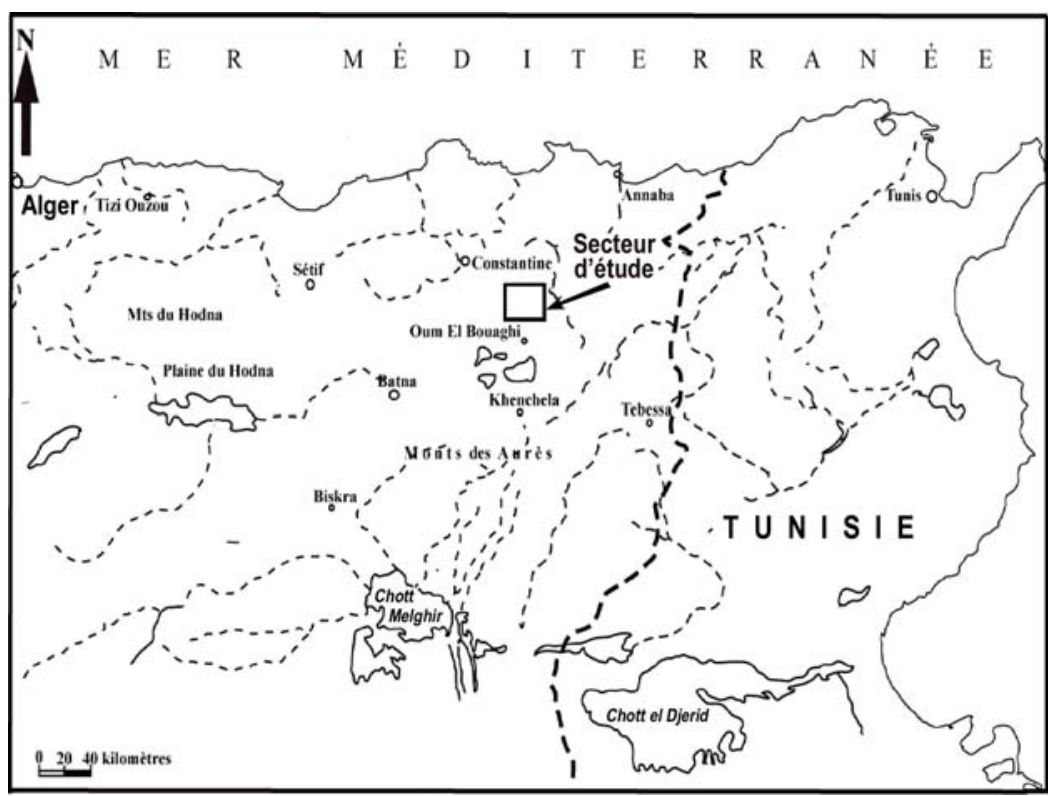

Fig. 1. Situation géographique de la zone d'étude.

Fig. 1. Location of the study area.

étude physico-chimique de l'eau de ces mêmes stations, afin de connaître d'une part l'état actuel de la qualité de l'eau qui est fréquemment consommée par les populations, et de connaître également la faune stygobie de la région afin de rechercher ensuite une possible corrélation entre la composition des zoocénoses et les caractéristiques de l'eau de ces puits et sources, comme celle qui a déjà été mise en évidence dans d'autres pays africains comme le Maroc ou le Cameroun.

\section{MATÉRIEL ET MÉTHODES}

\subsection{Zone d'étude}

Les stations étudiées sont situées au nord-ouest de la ville d'Oum-ElBouaghi, dans la partie sud-ouest de la plaine de Tamlouka (Fig. 1). Cette région est comprise entre l'Atlas Tellien au Nord et la chaîne de Sellaoua au Sud; elle se trouve dans le prolongement oriental des hauts-plateaux et est caractérisée par un bioclimat de type semi-aride, à été chaud et à hiver tempéré.

Des études géologiques réalisées dans les régions nord-orientales de l'Algérie il ressort que la plaine de Tamlouka et en particulier la « région de Guelma » se trouve au point de rencontre de trois domaines structuraux : celui du Sellaoua, et ceux des nappes néritiques du Constantinois et du Tellien. La plaine de Tamlouka est constituée essentiellement par un remplissage d'alluvions fluviatiles, d'âge plio-quaternaire, qui reposent sur des formations du 


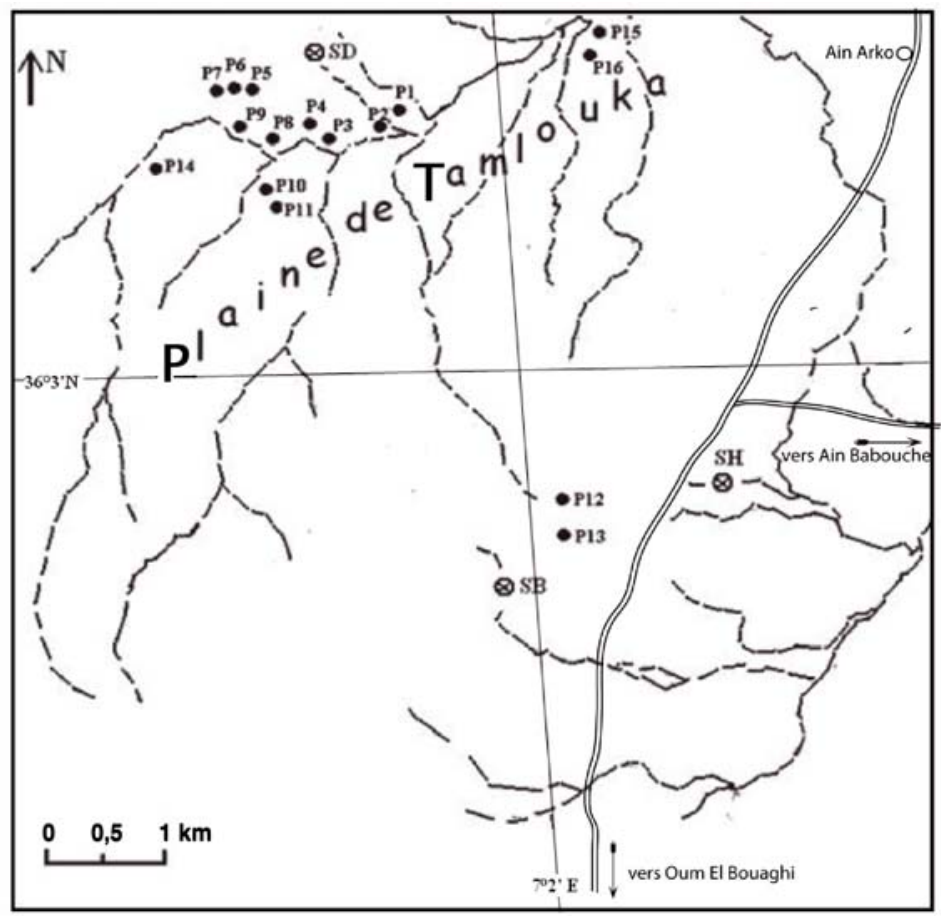

Fig. 2. Localisation géographique des 18 stations échantillonnées, au Nord de la ville d’Oum-ElBouaghi et au Sud-Est de Constantine. P1 : Puits $n^{\circ} 1$; SD : Source D.

Fig. 2. Geographical location of the 18 sampled stations, North of Oum-El-Bouaghi city and South-Est of Constantine city. $\mathrm{P} 1$ : Well $\mathrm{n}^{\circ} 1$; $\mathrm{SH}$ : Spring water $\mathrm{H}$.

Crétacé supérieur. La nappe phréatique de Tamlouka, au sud de l'Atlas Tellien, est donc contenue dans des formations de remplissage récentes. L'écoulement de cette nappe s'effectue le plus souvent, comme celui des principaux cours d'eau superficiels, dans le sens Sud-Ouest Nord-Est (Fig. 1). Les contacts tectoniques entre les différentes formations aquifères profondes se trouvent ainsi masqués par une puissante couverture alluvionnaire et restent encore très peu connus en dépit des tentatives récentes de leur exploration par des méthodes géoélectriques (Hemila \& Kowalski, 2002).
Une vingtaine de stations ont fait l'objet périodiquement d'une étude faunistique et physico-chimique, durant deux années. Les seize puits et les deux sources qui ont été retenus au cours de la présente étude sont localisés sur la figure 2. La profondeur de la nappe phréatique sous la surface du sol varie selon les secteurs en fonction du relief; elle est relativement grande vers l'amont (entre 9 et $12 \mathrm{~m}$ ) et diminue progressivement vers l'aval hydraulique de la nappe (entre 5 et $8 \mathrm{~m}$ ) dans l'ensemble des 16 puits et 2 sources qui ont été étudiés. Le choix des stations s'est porté 
essentiellement sur les puits anciens dont l'eau sert traditionnellement à divers usages agricoles et domestiques, y compris parfois d'eau de boisson, pour la population, en particulier pour un certain nombre de ces puits ( $\mathrm{P} 10$, $\mathrm{P} 11, \mathrm{P} 12, \mathrm{P} 14$ et P15) qui sont relativement protégés des apports extérieurs par une margelle ou par un couvercle, parfois par les deux. On doit enfin mentionner que l'ensemble du secteur d'étude est situé dans une zone agro-pastorale (céréaliculture et élevage ovin) et que dans le nord-ouest de la zone, où se trouve notamment l'ensemble des puits $P 1$ à $P 9$, des cultures plus denses que dans le reste de la région sont l'objet - et surtout ont été l'objet dans le passé - d'apports de pesticides et de fertilisants parfois importants, causant très probablement un impact non négligeable sur la nappe phréatique. Le puits P13 est un peu particulier car bien que situé relativement au Sud et pourvu d'un couvercle, il n'est pratiquement plus utilisé et reçoit encore des apports polluants provenant d'une ancienne moto-pompe à gasoil restée tout au bord du puits.

\section{2 Échantillonnage de la faune et techniques d'analyse de l'eau}

Des prélèvements mensuels de la faune et de l'eau ont été effectués dans les puits à l'aide d'un filet phréatobiologique, conçu selon le modèle d'abord mis au point par Cvetkov (1968), et légèrement modifié ultérieurement par Mittelberg et par Boutin. L'efficacité de ce mode de prélèvement a été reconnue (Boutin \& Boulanouar, 1983), notamment pour la capture de la faune planctonique et nectonique.
Cette technique a cependant été complétée par l'utilisation de pièges appâtés qui sont souvent plus efficaces pour la récolte de la faune rampante, interstitielle ou fouisseuse, en particulier pour certains Crustacés comme les Isopodes (Boutin \& Boulanouar, 1983). La faune des sources a été échantillonnée à l'aide d'une pompe Bou-Rouch (Bou \& Rouch, 1967) utilisée près du point d'émergence. La faune récoltée lors de chaque prélèvement a été fixée sur place au formaldéhyde à $8 \%$ puis extraite, triée, dénombrée et enfin identifiée au laboratoire. Le peuplement d'origine épigée, formé le plus souvent par des larves d'insectes immatures, et qui ne caractérise pas la nappe phréatique, a été déterminé seulement au niveau de la famille.

Les caractéristiques physicochimiques de l'eau telle que température, $\mathrm{pH}$, conductivité et oxygène dissous ont été mesurées sur le terrain à l'aide d'appareils de mesure portables WTW. Les ions indicateurs de pollution organique - d'une part les ions nitrates, nitrites et ammonium, et d'autre part les ions orthophosphates - ainsi que, la dureté calcique et la dureté magnésienne et les teneurs en ions sodium, potassium, sulfates et chlorures ont été dosés au laboratoire, conformément aux normes AFNOR (1983) et aux méthodes préconisées par Rodier (1984).

\subsection{Analyses statistiques}

Des analyses en composantes principales (ACP) ont été réalisées sur les données physico-chimiques (valeurs moyennes de l'ensemble des mesures 
mensuelles) figurant au tableau I puis sur les données biologiques caractérisant les différentes stations (Tab. II). Le traitement des données a été effectué à l'aide du logiciel SPSS 10.0.5 (SPSS, 1994). Chacune de ces ACP a été complétée par une classification hiérarchique qui a permis de délimiter objectivement les groupes de stations et d'obtenir ainsi une typologie.

\section{RÉSULTATS ET DISCUSSION}

\subsection{Physico-chimie des eaux}

Au cours de leur circulation souterraine et de leur séjour dans l'aquifère, les eaux phréatiques se chargent, au contact des différents terrains traversés, d'un certain nombre d'éléments chimiques solubles, chlorures, carbonates, sulfates et autres. Ces éléments sont d'origine géologique le plus souvent; ils peuvent cependant parfois être d'origine anthropique et sont alors susceptibles d'affecter sensiblement la qualité de l'eau de la nappe et par suite les caractéristiques de l'écosystème.

L'examen des résultats obtenus (Tab. I) montre que la qualité des eaux souterraines est très variable d'un puits à l'autre et d'une source à l'autre. L'ACP sur les valeurs moyennes de ces facteurs physico-chimiques montre que les deux premiers axes $\mathrm{F} 1$ et F2 représentent ensemble $53 \%$ de l'inertie totale. Le premier axe semble exprimer la qualité de l'eau, en opposant principalement les caractéristiques de l'eau de bonne qualité du côté négatif à des marqueurs de pollution (principalement les teneurs élevées en phosphates et en ions azotés) du côté positif. Le second axe distingue plutôt les facteurs déterminants de la minéralisation des eaux plutôt calco-magnésiennes du côté positif qu'à tendance séléniteuse du côté négatif. Cependant si les projections d'une demi-douzaine de facteurs sont rassemblées du côté positif du premier axe (Fig. 3A) celles des autres facteurs sont plutôt dispersées sur le plan des deux premiers axes, ce qui indique une relative indépendance des divers caractères mesurés. La typologie des stations issue de cette analyse, et confirmée par une classification hiérarchique, permet de distinguer clairement quatre groupes de stations (Fig. 3B) qui diffèrent par la qualité physico-chimique de leurs eaux :

groupe 1 : il isole la source $\mathrm{SH}$, qui présente des eaux de très bonne qualité, caractérisées par une minéralisation faible et un taux très faible voir nul en ions indicateurs de la pollution organique;

groupe 2 : il ne contient que la source SD qui présente également une eau de bonne qualité, mais relativement plus minéralisée par comparaison avec celle de la source $\mathrm{SH}$. Cette différence entre la qualité de l'eau produite par de ces deux sources peut certainement s'expliquer par des différences locales de la composition des roches aquifères, les sources étant localisées dans deux vallées distinctes distantes de quelque $5 \mathrm{ki}$ lomètres seulement mais surtout dans 2 bassins hydrographiques différents, sur les versants opposés d'une zone collinaire la Chebket es Sellaoua, allongée dans le sens sud-ouest nord-est. En fait SD est dans les alluvions récentes de 


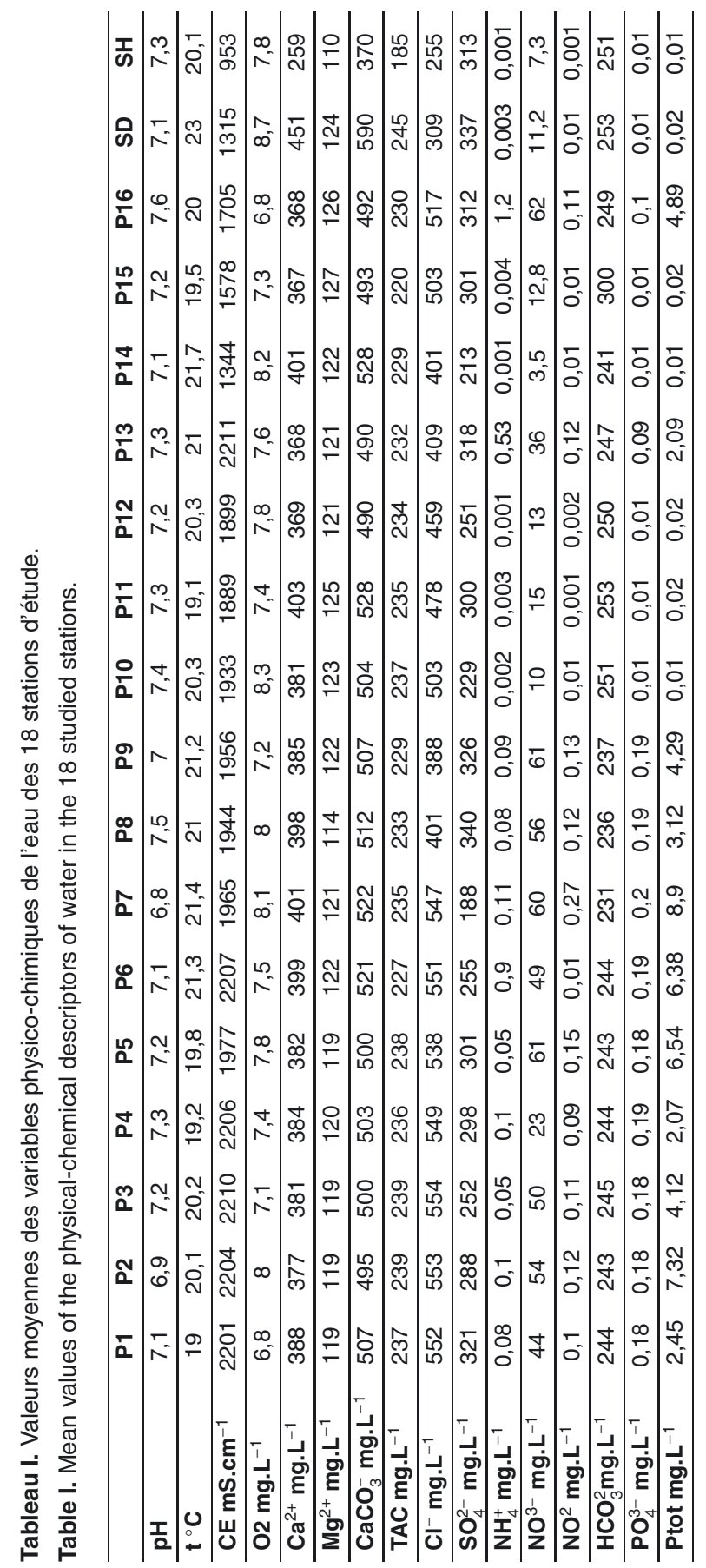




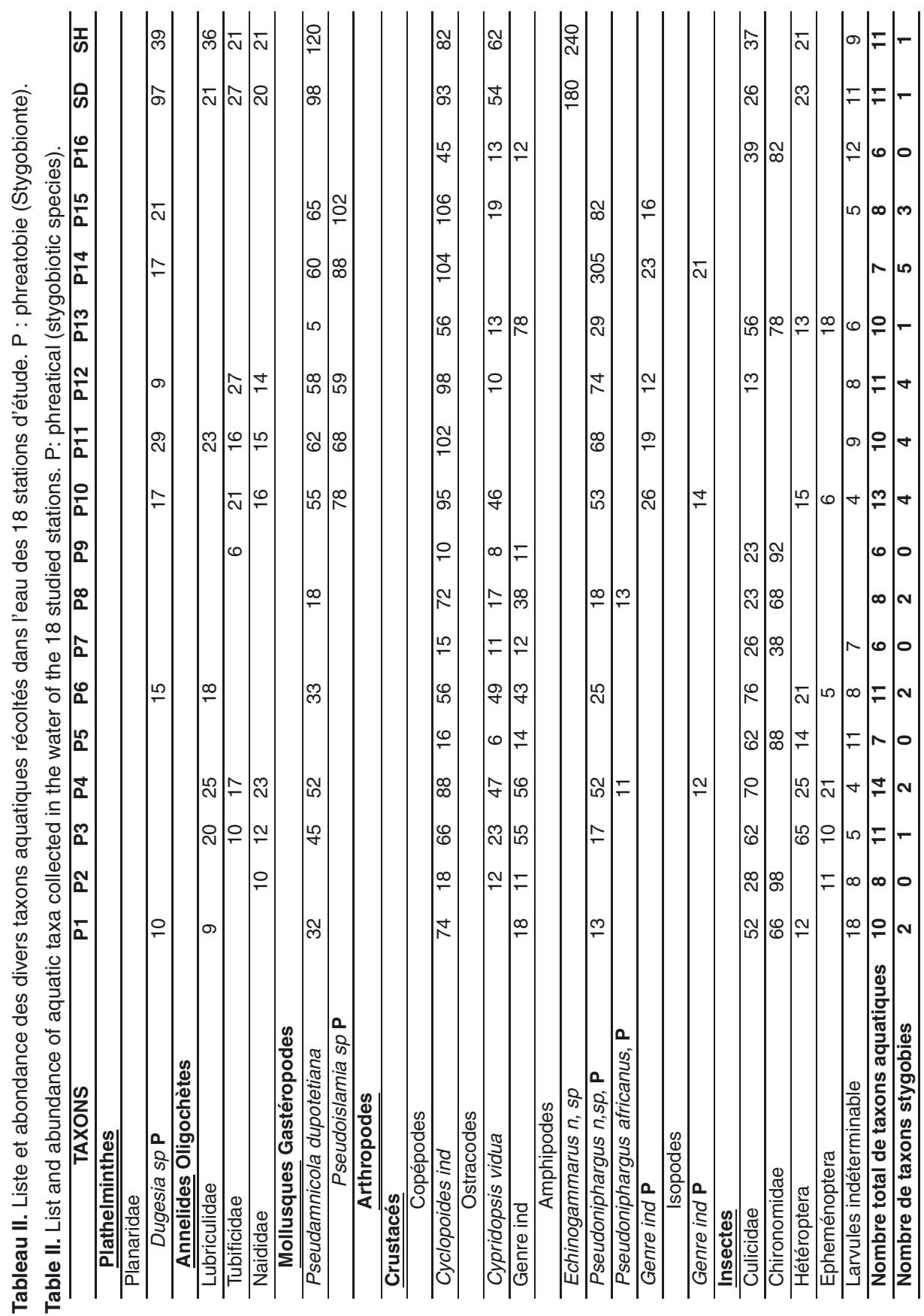




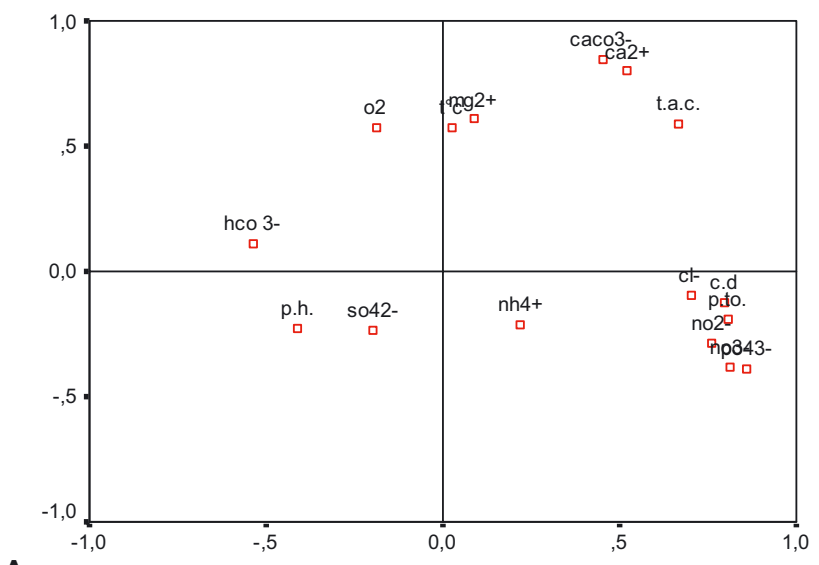

A

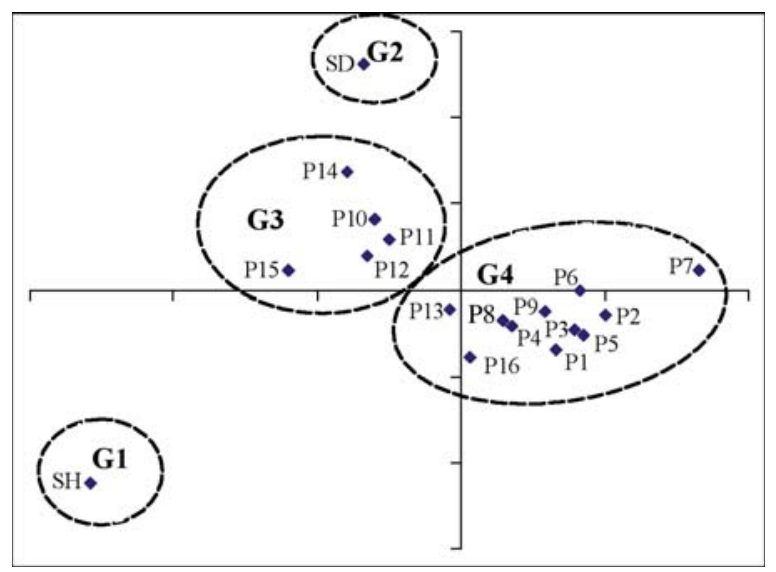

B

Fig. 3. A : Projections des variables physico-chimiques de l'eau figurant dans le tableau II, sur le plan des deux premiers axes factoriels de l'ACP (axe 1 horizontal et axe 2 vertical). B : Projection des 4 groupes de stations, décrites par leurs caractéristiques physico-chimiques, sur le plan des deux premiers axes de l'ACP (axe 1 horizontal et axe 2 vertical).

Fig. 3. A: Projections of physical-chemical variables of water from the 18 stations (as given in Tab. I on the plan of the two first axes of the PCA (axis 1 horizontal and axis 2 vertical). B: Projections of the four groups of studied stations based on the physicochemical characteristics of their water, on the plan of axis 1 (horizontal) and axis 2 (vertical) of the PCA. 
la plaine de Tamlouka alors que $\mathrm{SH}$ est dans les terrains plus anciens constituants les écailles des Sellaoua ;

groupe 3 : il réunit cinq puits, $\mathrm{P} 10$, $\mathrm{P} 11, \mathrm{P} 12, \mathrm{P} 14$ et $\mathrm{P} 15$, qui sont tous des puits protégés des apports éoliens par des couvercles et situés assez loin des lieux habités. Aucun indice de pollution - ni organique ni minérale - n'a été détecté ;

groupe 4: ce groupe réunit l'ensemble des 11 autres puits : $\mathrm{P} 1$, P2, P3, P4, P5, P6, P7, P8, P9, $\mathrm{P} 13$ et $\mathrm{P} 16$, dont les eaux sont de qualité médiocre à mauvaise. Elles sont en effet plus riches en ions indicateurs de pollution organique, dont les teneurs dépassent souvent les normes fixées par l'OMS. Les nitrates par exemple sont présents à des concentrations moyennes qui vont de 23 à $61 \mathrm{mg} \cdot \mathrm{L}^{-1}$ selon les stations; leur concentration moyenne atteint par exemple $50 \mathrm{mg}$ par litre dans le puits P3, mais même dans ce puits elle dépasse souvent cette valeur seuil. Enfin dans les 6 stations P2, P5, $\mathrm{P} 7, \mathrm{P} 8, \mathrm{P} 9$ et $\mathrm{P} 16$, même les valeurs moyennes sont supérieures à ce seuil puisqu'elles sont généralement proches de $60 \mathrm{mg}$ par litre. Les nitrites sont toujours présents à des concentrations qui vont de 0,1 à $0,27 \mathrm{mg} \cdot \mathrm{L}^{-1}$ et même les ions ammonium sont détectables dans ces puits en dépit du fait que l'eau est aussi oxygénée (avec 6,8 à $8 \mathrm{mg} \cdot \mathrm{L}^{-1}$ d'oxygène dissous) que dans les stations du groupe 3 (voir Tab. I). Les eaux de ces puits présentent donc un risque potentiel pour la santé des populations locales qui les consomment.
De ces résultats et des observations de terrain, il ressort que, dans l'ensemble de la région d'étude, les eaux sont fortement minéralisées, avec notamment des valeurs élevées de la dureté totale, de la salinité et des teneurs en sulfates, tous ces ions provenant des roches calcaires ou salines traversées par les eaux souterraines; cela ne représente certainement pas un réel danger pour les utilisateurs. Cependant si certaines stations révèlent une pollution organique légère, d'autres en revanche (toutes celles du groupe 4), montrent une pollution sérieuse. Les stations dont l'eau est généralement de bonne qualité se trouvent localisées loin des sources de pollution et il apparaît sur le terrain que la qualité de l'eau se dégrade lorsque l'on se rapproche des zones d'habitation. Les causes principales de pollution de l'eau de ces puits sont visiblement en rapport avec les rejets d'eaux usées brutes qui circulent dans des petits canaux ou "seguias " utilisées souvent pour l'irrigation, ou plus simplement pour évacuer l'eau usée loin des habitations; dans tous les cas ces eaux usées peuvent s'infiltrer jusqu'à la nappe phréatique. Une seconde cause de pollution semble liée à la présence d'accumulations de fumier et parfois de purin, provenant des bergeries et des étables. Les fumiers sont périodiquement lessivés par l'eau des pluies qui entraîne vers la nappe bactéries et substances solubles.

\subsection{Diversité du peuplement}

La faune récoltée contient à peine 20 taxons qui figurent dans le tableau II. Cependant la liste des 
espèces aquatiques rencontrées est encore provisoire et certainement incomplète car de nombreux taxons, comme les Amphipodes Gammaridae ou les Annélides Oligochètes n'ont pas encore pu être déterminés au niveau de l'espèce, et il est probable qu'ils renferment plusieurs espèces distinctes, voire des genres dont la systématique, ainsi que la signification écologique méritera d'être précisée. Enfin des Némathelminthes parfois présents dans certains prélèvements, mais difficiles à identifier n'ont pas été non plus pris en compte. Parmi ces espèces, certaines sont d'origine superficielle; ce sont par exemple des insectes, des arachnides, des mollusques ou certains crustacés qui peuvent se rencontrer ailleurs, dans les eaux de surface, cours d'eau, mares ou lacs. D'autres, en revanche, sont d'origine souterraine, elles vivent normalement dans l'eau de la nappe phréatique, et peuvent se déplacer entre les grains de sable et les graviers qui constituent l'aquifère (ce sont les espèces interstitielles au sens strict), ou encore en creusant des galeries dans le sédiment, donc en déplaçant les grains, (ce sont alors des espèces fouisseuses). Ces espèces souterraines (ou stygobies) sont les plus intéressantes en raison de leur plus grande sensibilité à la qualité des eaux. Par leur présence, ces espèces renseignent donc sur l'état de l'eau de la nappe phréatique, alors que la présence des espèces épigées, généralement plus résistantes dépend plutôt des caractères hydrologiques du puits, de sa morphométrie, de son aménagement et de sa protection en surface, enfin de facteurs aléatoires de dispersion tels que l'arrivée d'insectes aériens venus pondre dans le puits, ou encore d'oiseaux, de batraciens ou autres animaux vecteurs venant occasionnellement dans les puits, ou parfois transportés par l'homme ou par le vent. II résulte de ces aléas et du grand nombre de ces espèces épigées que la projection des taxons sur le plan factoriel des deux premiers axes de l'ACP (Fig. 4A) ne présente pas de structuration très significative. Toutefois l'axe 1 pourrait, là encore, être indirectement en rapport avec la qualité de l'eau, dans la mesure où on observe que les taxons les plus communs et qui sont connus pour bien résister à la pollution de l'eau (tels que les Culicidés ou les Chironomidés) s'opposent, du côté négatif, aux taxons souterrains (comme les crustacés amphipodes stygobies du genre Pseudoniphargus, ou les mollusques gastéropodes Pseudoislamia) qui sont plus sensibles à la pollution et qui se trouvent du côté des coordonnées positives.

La projection des stations (décrites par leur faune) sur le plan des deux premiers axes factoriels de l'ACP (Fig. 4B) permet de distinguer clairement trois grands groupes de stations :

groupe I: il réunit les deux sources $\mathrm{SD}$ et $\mathrm{SH}$ qui présentent la même composition faunistique, aussi bien en taxons stygobies qu'épigés. Ces sources se distinguent des autres stations par une proportion plus élevée de taxons épigés qui peut être due aux caractéristiques de ces écosystèmes largement ouverts et sans protection, par comparaison avec les puits. Ils sont donc plus favorables à l'installation des espèces épigées ; 
AA

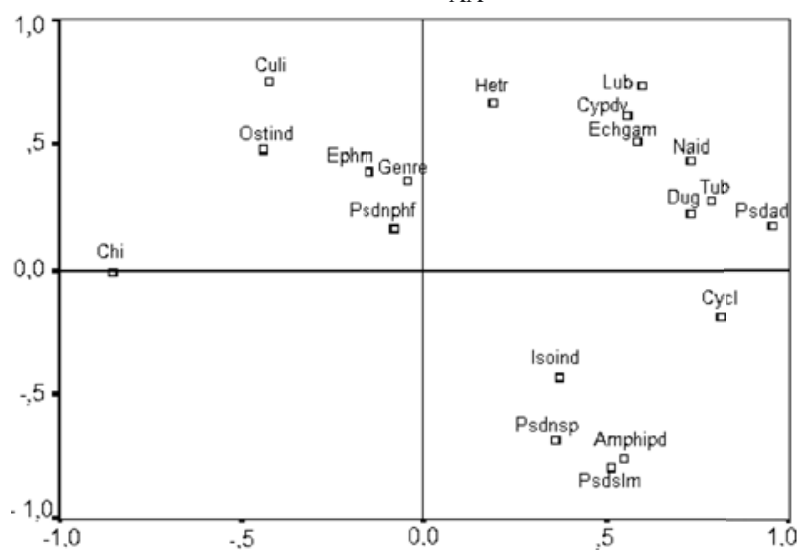

$\mathbf{A}$

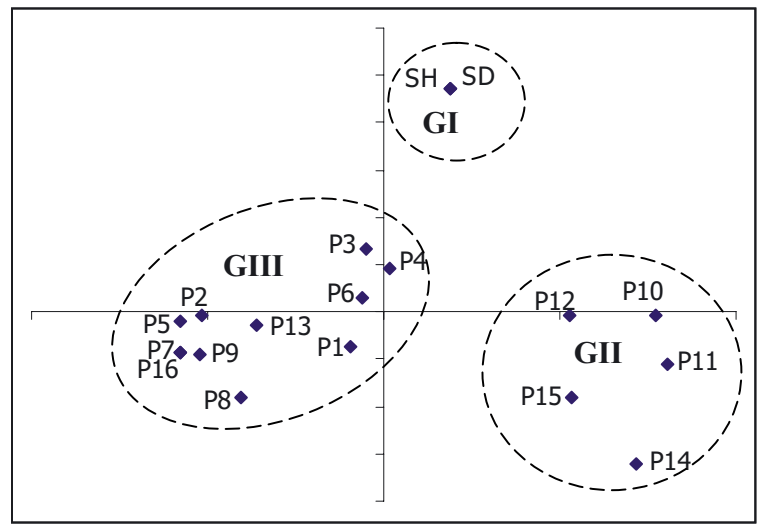

B

Fig. 4. A : Projections des taxons récoltés au cours de l'étude sur le plan des deux premiers axes de l'ACP (axe 1 horizontal et axe 2 vertical). Dug : Dugesia sp.; Lub : Lumbriculidae, Tub : Tubificidae; Naid : Naididae; Psdadp : Pseudomnicola dupotetiana; Psdislm : Pseudoislamia sp.; Cycl : Cyclopoides; Cypdv : Cypridopsisvidua; Ostind : Ostracodes indéterminés; Echgam : Echinogammarus sp.; Psdnsp : Pseudoniphargus sp.; Psdnphf : Pseudoniphargus africanus; Amphipd : Amphipode non determine; Isoind : Isopode non determiné; Culi : Culicidae; Chi : Chironomidae; Hetr : Heteroptera; Ephm : Ephemeroptera; Genre : larvules d'Insecte non determinables. B : Projections des stations, décrites par leur faune, sur le plan des deux premiers axes factoriels de l'ACP (axe 1 horizontal et axe 2 vertical).

Fig. 4. A: Projections of sampled taxa on the plan of the two first axes of the PCA (axis 1 horizontal and axis 2 vertical). Names of taxa like above. B: Projections of stations, described by their fauna (from Tab. II), on the plan of the two first axes of the PCA (axis 1 horizontal and axis 2 vertical). 
groupe II : ce groupe comprend les cinq puits P10, P11, P12, P14 et $P 15$. Chacun de ces puits a livré au total de 7 à 13 espèces aquatiques, avec un nombre moyen de 9,8 et dans chacune des 5 stations, on note la présence de 4 ou 5 espèces stygobies, 4,4 en moyenne. Le nombre des espèces stygobies représente en moyenne pour les 5 puits de ce groupe, plus de $43 \%$ de la richesse spécifique globale;

groupe III : il comprend les 11 puits, P1, P2, P3, P4, P5, P6, P7, P8, $\mathrm{P} 9, \mathrm{P} 13$ et P16. Dans ces stations la richesse spécifique globale n'est en moyenne que de 8,8 , mais ce qui est remarquable c'est que la faune stygobie est soit totalement absente (dans 5 des stations) soit représentée par 1 ou 2 espèces seulement, avec un nombre moyen d'espèces stygobies de l'ordre de 0,9 (donc significativement plus faible que dans les puits du second groupe où il était de 4,4$)$. Le nombre des espèces stygobies représente en moyenne pour les 11 puits de ce groupe, moins de $9 \%$ de la richesse spécifique globale. On doit rappeler ici que presque tous ces puits sont à ciel ouvert, sans protection et plus que les autres exposés aux pollutions locales.

\section{DISCUSSION ET CONCLUSIONS}

Les investigations rapportées dans cet article constituent une première étape d'une approche écologique régionale orientée vers la recherche des rapports entre la faune et la qualité de l'eau. Elle mérite d'être poursui- vie par une revue systématique de la faune aquatique souterraine récoltée dans la région d'étude, comportant pour chaque taxon des commentaires taxonomiques et biogéographiques en plus des caractères écologiques.

\subsection{Conclusion générale}

On doit d'abord constater que toutes les stations prospectées dans cette région du Nord-Est algérien ont livré un bon nombre d'espèces aquatiques épigées (en moyenne 9,33 espèces) nettement plus élevé que celui des espèces souterraines (1,88 en moyenne) et que ces dernières étaient parfois absentes (Fig. 5). Ce résultat toutefois n'a rien de surprenant car on sait depuis longtemps que les sources comme les puits sont des écotones montrant un mélange de deux groupes d'espèces, les unes épigées et les autres endogées, cohabitant dans le même biotope comme cela a maintes fois été souligné par ceux qui se sont intéressés aux puits dans une optique biocénotique (Vandel, 1964 ; Dalmas, 1972, 1973). Observée en Europe d'abord, cette caractéristique des puits ordinaires avait déjà été vérifiée dans différentes régions du monde, en particulier au Maghreb à l'occasion des recherches menées par Boulanouar (1986, 1995), Boulal (1988, 2002), Idbennacer (1990), Fakher El Abiari (1999), Ait Boughrous et al. (2007) dans diverses régions du Maroc, et ce au Nord comme au Sud de la chaîne atlasique. La sensibilité particulière à la pollution des espèces aquatiques souterraines, comparées à celle des espèces épigées avait également été observée (Boutin, 1984; Boutin 


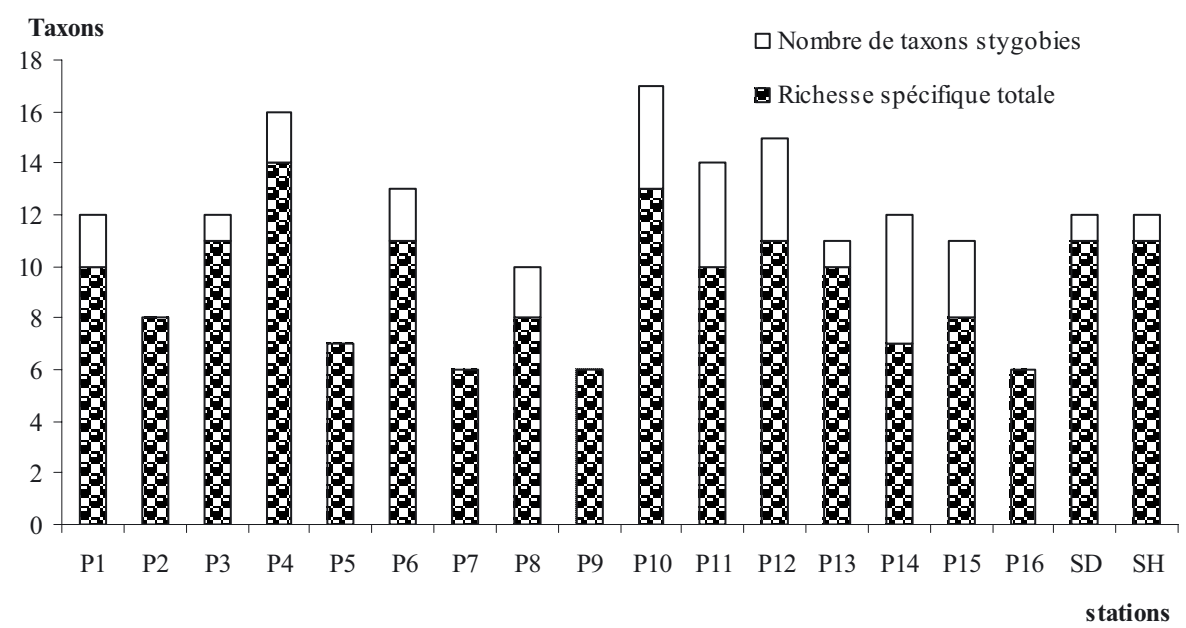

Fig. 5. Variabilité inter-stationnelle de la richesse taxonomique totale (en gris foncé), et de la richesse en espèces stygobies (en blanc) dans l'ensemble des 18 stations étudiées.

Fig. 5. Total taxonomic richness of the 18 studied stations (bottom, in grey) and richness of the stygobiotic fauna (in white, above).

et al., 1995) et l'idée avait été émise de la possibilité d'utiliser ces espèces comme indicatrices (par leur présence) de la qualité de l'eau d'un puits ou d'une nappe, ou au contraire (par leur absence) de la pollution de la nappe (Boutin, 1987; Boutin \& Dias, 1987). Les résultats de la présente étude viennent donc utilement confirmer et de façon très intéressante les résultats précédents, leur conférant ainsi un caractère plus général.

\subsection{Relation entre la biodiversité faunistique et la qualité physico-chimique de l'eau}

La recherche d'une typologie des stations grâce à des ACP a conduit à un résultat remarquable : que l'on utilise comme descripteurs les caractéristiques physico-chimiques de l'eau (Fig. 3B) ou au contraire les espèces aquatiques présentes dans chaque station (Fig. 4B), les résultats sont identiques. Dans les deux cas on observe en effet une première séparation dans l'ensemble des stations entre les sources d'une part et les puits d'autre part; certes cela n'a rien de surprenant, les conditions écologiques qui règnent à l'émergence d'une source et celle qui prévalent dans l'eau au fond d'un puits étant bien différentes. Mais il est plus remarquable que l'ensemble des 16 puits se trouve dans les deux cas séparé en deux groupes de 5 et 11 puits respectivement, qui sont exactement les mêmes dans les deux analyses. Le groupe 2 de la seconde ACP réunit les mêmes puits ( $\mathrm{P} 10$ à $\mathrm{P} 12$ plus $\mathrm{P} 13$ et P14) que ceux du groupe 3 de la première analyse. De même les 11 autres puits - les stations dont l'eau est douteuse ou franchement polluée se trouvent réunis dans un même ensemble dans les deux analyses. 
Dans les puits les plus pollués (le groupe de 11 puits), la faune est moins diversifiée et surtout la faune stygobie est soit absente soit réduite à 1 ou 2 espèces au maximum. À l'inverse, dans les 5 puits de l'autre groupe où l'eau est de bonne qualité, la diversité taxonomique est en moyenne plus élevée et surtout la faune stygobie est représentée par 4 ou 5 espèces différentes.

Force est de conclure que, dans la zone d'étude, comme dans les autres régions où ont travaillé les auteurs marocains déjà cités, les descripteurs biologiques sont de bons indicateurs de la qualité d'un milieu aquatique souterrain. Leur intégration, à l'avenir, dans les bilans d'évaluation de la qualité de l'eau serait donc un outil supplémentaire permettant d'apprécier globalement l'état de l'écosystème, afin de pouvoir en assurer la surveillance et si besoin la protection de l'environnement aquatique souterrain. Et cela d'autant plus qu'un échantillonnage de la faune aquatique des puits et des sources est beaucoup moins onéreux qu'une série d'analyses physico-chimiques suivie par une série d'analyses bactériologiques. II ne serait donc pas déraisonnable de proposer qu'une prospection faunistique de l'eau des puits soit préconisée à une large échelle dans les zones rurales où un contrôle classique de la qualité de l'eau, physico-chimique et bactériologique général serait trop onéreux voire impossible. Bien évidemment cela demanderait le concours dans chaque région de quelques zoologistes mais les analyses de laboratoire qui ont un coût important et dont la liste est toujours incomplète, pourraient alors être envisagées seule- ment lorsque l'étude faunistique aurait préalablement révélé un problème de qualité de l'eau. D'ailleurs il faut noter que tout récemment (après que la présente étude ait été réalisée), plusieurs auteurs allemands (Steube et al., 2009) ont souligné l'importance de la prise en compte de la dimension écologique des divers aquifères en soulignant l'intérêt de la faune aquatique des eaux souterraines - à côté des analyses physico-chimiques et de la bactériologie - pour évaluer l'état des réserves, suivre son évolution et orienter les stratégies de protection.

Dans la région étudiée au Nord de la Plaine de Tamlouka, il serait cependant utile de compléter les résultats de ce travail par une étude bactériologique comparée de l'eau dans l'ensemble des 18 stations considérées, afin d'apporter une preuve supplémentaire de la validité de nos conclusions. Cela fait d'ailleurs partie des projets du Laboratoire d'Hydrobiologie de l'Institut des sciences de la nature de l'université d'Oum-El-Bouaghi. Mais il faut savoir que, dans les rares cas où cela fut réalisé, comme dans l'étude de Boutin \& Dias (1987) déjà ancienne, les résultats de la bactériologie, de la physicochimie et de l'analyse de la biodiversité étaient concordants, la charge organique polluante décelée par les analyses de l'eau étant accompagnée d'une présence et d'une diversité bactérienne dangereuse. Mais l'approche biocénotique à elle seule permet déjà de caractériser l'état de la nappe et plus précisément des points d'eau utilisés par la population.

II est donc souhaitable d'étendre le champ des investigations visant aussi à connaître la stygofaune de l'Est algérien notamment - en multipliant le 
nombre des stations, en répétant plusieurs fois les prélèvements, et en multipliant, si besoin, le nombre de paramètres mesurés (Sullivan, 1982). En effet l'examen de la faune d'un puits nouvellement échantillonné par un stygobiologiste permettra, à peu de frais, de ranger ce puits soit dans le groupe 2 de la seconde ACP, donc parmi les puits peu ou pas pollués, non suspects a priori (Fig. 4B), soit au contraire dans le groupe 3 de la même ACP, celui des puits pollués et donc très suspects. Les analyses bactériologiques et physicochimiques de l'eau des puits pollués peuvent venir ensuite mais moins nombreuses elles occasionneront une moindre dépense. Et sans en attendre les résultats, il est tout à fait possible, dès que l'analyse faunistique signale des stations suspectes, de rechercher aux alentours les causes probables de la pollution, et de préconiser des mesures locales adaptées permettant d'y remédier. Ce type de recherche, orienté vers la connaissance de la biodiversité de la faune des puits, notamment dans les régions rurales où l'approvisionnement en eau des populations est dépendant des puits, présente donc d'abord un intérêt appliqué à l'amélioration de la santé publique (Boutin, 1987).

II faut mentionner également que de telles recherches présentent un intérêt fondamental et patrimonial, dans la mesure où elles permettent de découvrir une part cachée de la biodiversité de chaque région étudiée, donc une part du patrimoine naturel national. L'endémisme des espèces animales est beaucoup plus important dans les milieux souterrains (qu'ils soient terrestres ou aquatiques) que dans les milieux de surface comparables. Nous avons par exemple constaté qu'un type de Crustacés Isopodes stygobies du genre Typhlocirolana, représenté par plusieurs espèces dans de nombreuses régions d'Algérie (Monod, 1930), y compris dans la région de Biskra un peu plus au Sud, semble totalement absent dans la zone que nous avons étudiée, mais qu'en revanche les Amphipodes semblent particulièrement bien représentés avec deux espèces différentes de Pseudoniphargus et probablement un genre nouveau d'Amphipodes, encore indéterminé à ce jour. II est certain que l'étude taxonomique de ces espèces qui n'avaient jamais été signalées dans la région au préalable et qui sont très probablement nouvelles pour la science, sera d'un grand intérêt, de même que l'explication de leur présence ou de leur absence à certains endroits, lorsque l'étendue de leurs aires de répartition sera connue de façon plus complète (Boutin, 1993). Ces considérations ne peuvent que pousser à étendre le champ des prospections.

L'étude des puits de l'Est algérien présente donc à la fois un intérêt appliqué très important puisqu'il concerne la santé publique, et par ailleurs un intérêt fondamental tout aussi important puisqu'elle conduit vers des recherches de taxonomie zoologique et de biogéographie historique, avec la découverte d'espèces nouvelles pour la science.

\section{REMERCIEMENTS}

Nous tenons à remercier ici les autorités de l'université Larbi Ben M'hidi d'Oum-ElBouaghi (Algérie) qui ont autorisé et facilité le travail de terrain des premiers auteurs, 
ainsi que les deux correcteurs anonymes qui ont révisé le manuscrit avant son acceptation, et qui ont contribué par leurs critiques constructives à l'amélioration du texte et à l'enrichissement de la bibliographie en suggérant notamment l'addition d'une référence récente et importante.

\section{RÉFÉRENCES BIBLIOGRAPHIQUES}

AFNOR, 1983. Association française de normalisation : Eaux, méthodes d'essai. $2^{\mathrm{e}}$ édition, Paris.

Aït Boughrous A., Yacoubi-Khebiza M., Boulanouar M., Boutin C. \& Messana G., 2007. Qualité des eaux souterraines dans deux régions arides du Maroc : impact des pollutions sur la biodiversité et implications paléogéographiques. Environmental Technology 28 : 1299-1315.

Belaidi-Aliane N., 2004. Rôle du milieu hyporhéique dans le fonctionnement de l'écosystème Oued. Dynamique de la faune hyporhéique à l'aval d'un barrage pollué. Thèse doctorat d'État, Univ. Abou Bekr Belkaid, Tlemcen, 78 pp.

Bosca C., 2002. Groundwater law and administration of sustainable development. Mediterranean Magazine, Science, Training and Technology 2 : 13-17.

Botosanéanu L., 1971. Observation sur la faune aquatique hypogée des monts du Banat, Roumanie. Trav. Inst. Spéol. E. Racovitza 10 : 123-166.

Botosanéanu L., 1986. Stygofauna mundi, a Faunistic, Distributional, and Ecological Synthesis of the World Fauna inhabiting Subterranean Waters (including the Marine Intertitial). E. J. Brill Ed. (Leiden), 740 pp.

Bou C., 1974. Recherches sur les eaux souterraines : les méthodes de récolte dans les eaux souterraines interstitielles. Ann. Spéléol. 29 (4) : 611619.

Bou C. \& Rouch R., 1967. Un nouveau champ de recherches sur la faune aquatique souterraine. C.R. Acad. Sc. Paris. 265 : 369-370.

Boulal M., 1988. Recherches écologiques sur la faune aquatique des puits de la région de Tiznit (Anti-Atlas occidental, Maroc). Thèse de $3^{\mathrm{e}}$ cycle, Fac. Sc. Marrakech, $228 \mathrm{pp}$.

Boulal M., 2002. Recherches phréatobiologiques dans le Souss et les régions voisines du Maroc Occidental : Qualité de l'eau des puits, Biodiversité, Écologie et Biogéographie historique des espècess stygobies. Thèse de doctorat d'État, Fac. Sc. Marrakech, $425 \mathrm{pp}$.

Boulanouar M., 1986. Etude écologique comparée de quelques puits de la région de Marrakech. Impact des pollutions sur les zoocénoses des puits. Thèse de $3^{\mathrm{e}}$ cycle, Fac, Sc. Marrakech, $159 \mathrm{pp}$.

Boulanouar M., 1995. Faune aquatique des puits et qualité de l'eau dans les régions de Marrakech et des Jbilet. Statut et dynamique d'une population de Proasellus coxalis africanus (Crustacé Isopode) des Jbilet. Thèse de Doctorat d'Etat, Fac. Sc. Univ. Marrakech, $159 \mathrm{pp}$.

Bousfield E.L., 1958. Freshwater amphipod crustaceans of glaciated North America. Canad. Field Naturalist. 72 (2) : 55-113.

Boutin C., 1984. Sensibilité à la pollution et répartition de quelques espèces de crustacés phréatobies à Marrakech (Maroc occidental). Mém. Biospéol. 11 (38) : 55-64.

Boutin C., 1987. L'eau des nappes phréatiques superficielles, une richesse naturelle vitale mais vulnérable. L'exemple des zones rurales du Maroc. Sc. de l'eau 6 (3) : 357-365.

Boutin C., 1993. Biogéographie historique des Crustacés Malacostracés stygobies du Maroc. Thèse de Doctorat, Univ. Cl. Bernard Lyon I, France, 263 p.

Boutin C. \& Boulanouar M., 1983. Méthodes de capture de la faune stygobie. Expérimentation de différents types de pièges appâtés dans les puits 
de Marrakech. Bull. Fac. Sc. Marrakech $2: 5-21$.

Boutin C. \& Dias N., 1987. Impact de l'épandage des eaux usées de la ville de Marrakech sur la nappe phréatique. Bull. Fac. Sc. Marrakech (Section Sc. Vie) $3: 5-25$.

Boutin C. \& Idbennacer B., 1989. Faune stygobie du Sud de l'Anti-Atlas marocain : premiers résultats. Rev. Sc. de l'eau 2 : 891-904.

Boutin C. \& Messouli M., 1988a. Longipodacranconyx maroccanus n. gen., n. sp., nouveau représentant du groupe Metracranconyx (Crustaces, Amphipoda) dans les eaux souterraines du Maroc. Crustaceana. Suppl. $13: 156-271$.

Boutin C. \& Messouli M., 1988b. Metracranconyx gineti n. sp. D'une source du Haut-Atlas marocain et la famille des Metracranconyctidae $\mathrm{n}$. fam. (Crustacés Amphipodes stygobies). Vie et Milieu 38 (1) : 67-84.

Boutin C., Boulanouar M. \& YacoubiKhebiza M., 1995. Un test biologique simple pour apprécier la toxicité de l'eau et des sédiments d'un puits. Toxicité comparée, in vitro, de quelques métaux lourds et de l'ammonium, vis-àvis de trois genres de Crustacés de la zoocénose des puits. Hydroécol. Appl. 7 (1-2) : 91-109.

Coineau, N., 1971. Les Isopodes interstitiels, documents sur leur écologie et leur biologie. Mem. Mus. Nat. Hist. Nat. Paris. LXIV (A), 170 pp.

Coineau N. \& Boutin C., 1992. Biological processes in space and time : colonization, evolution and speciation in interstitial stygobionts. In The Natural History of Biospeleology, 423-451, A.I. Camacho ed. Monografias 7, Mus. Nat. Cie. Nat. C.S.I.C, Madrid, 680 p.

Culver D.C., 1982. Cave life : Evolution and Ecology. Harvard Univ. Press, Cambridge Massachusetts, 189 pp.

Culver D.C. \& White W.B. (Eds.), 2005. Encyclopedia of caves. Elsevier, Academic Press, Amsterdam, New York, $654 \mathrm{pp}$.
Cvetkov L., 1968. Un filet phréatobiologique. Bull. Inst. Zool. Mus. Sofia XXVII : 215-219.

Dalmas A., 1972. Contribution à l'étude des caractères physico-chimiques de l'eau et de la faune de quelques puits artificiels de la Provence. Thèse de doctorat, université de Provence, $160 \mathrm{p}$.

Dalmas A., 1973. Zoocénoses de puits artificiels en Provence. Ann. Spélol. 28 (3) : 517-522.

Danielopol D.L., Gibert J., Griebler C., Gunatilaka A., Hahn H.J., Messana G., Notenboom J. \& Sket B., 2004. Incorporating ecological perspectives in European groundwater management policy. Environ. Conservation. 31 (3) : 185-189.

Decu V., Juberthie C., Collignon B. \& Lebreton B., 2001 - «Algérie » In Encyclopaedia Biospeologica, Soc. Intern. Biospeleol., Bucarest et Moulis. 3 : 1477-1496.

Delamare Debouteville C., 1960. Biologie des eaux souterraines littorales et continentales. Hermann, Paris, 740 pp.

Dumont H.J., 1981. Cteniobathynella essameuri n. sp., the first representative of the Bathynellacea (Crustacea) in the central Sahara. Rev. Hydrobiol. Trop. 14 (1) : 59-62.

Fakher El Abiari A., 1999. Rôle des facteurs abiotiques dans la répartition de la faune aquatique souterraine du Maroc : Cas des Crustacés Péracarides stygobies. Thèse de doctorat National, Fac. Sc. Semlalia, Marrakech, 208 pp.

Gauthier H., 1928. Recherches sur la faune des eaux continentales de l'Algérie et de la Tunisie. Impr. Minerva. Alger : 419 pp. +3 Pl. et 6 Cartes H. T.

Gibert J., 1986. Écologie d'un système karstique jurassien : hydrogéologie, dérive animale, transits de matières et dynamique de la population de Niphargus (Crustacé, Amphipode). Mem. Biospeol. XIII (40) : 1-379.

Gibert J., Danielopol D.L. \& Stanford J.A. (Eds.), 1994. Groundwater Ecology. Academic Press, New York, 572 pp. 
Gibert, J., Mathieu J., Fournier F., (Eds.) 1997. Groundwater / Surface Water Ecotones : Biological and Hydrological Interactions and Management Options. Cambridge University Press, Cambridge, $246 \mathrm{pp}$.

Gunn J. (Ed.), 2004. Encyclopedia of Caves and Karst Science. Fitzroy Dearborn, Taylor and Francis Group, London, 902 pp.

Hemila M.L. \& Kowalski W.M., 2002. Synthèse géo-électrique appliquée à la caractérisation des contacts entre nappes de charriage et son apport hydrogéologique dans la Plaine de Tamlouka «Région de Guelma, Est Algérien ». Can. Geotec. Journ. 39 (3) : 725-737.

Holsinger J.R., 1972. The freshwater amphipod crustaceans (Gammaridae) of North America. Biota of freshwater ecosystems, Identification. Manual U. S. Environmental Agency 5 : 1-89.

Holsinger J.R., 1986. Zoogeographic patterns of North American subterranean amphipod crustaceans. In : R.H. Gore and K.L. Heck (Eds.), Crustacean Biogeography. Crustacean 3 : 85-106.

Holsinger J.R., 1994. Pattern and process in the biogeography of subterranean amphipod crustaceans. In D.C. Culver and J.R. Holsinger (Eds.), Biogeography of Subterranean Crustaceans : the effects of different scales. Hydrobiologia 287 (1) : 131-145.

Holsinger J.R., 2000. Ecological derivation, colonization, and speciation. In : H. Wilkens, D.C. Culver, \& W.F. Humphreys, (Eds.), Ecosystems of the World 30 : Subterranean Ecosystems. Elsevier. Amsterdam, New York, 399-415.

Idebenacer B., 1990. Recherches écologiques, biogéographiques et démographiques sur la faune aquatique souterraine de la région de Guelmin (Sud-ouest de l'Anti-Atlas marocain). Thèse de $3^{\mathrm{e}}$ Cycle, Fac. Sc. Semlalia. Marrakech, $118 \mathrm{pp}$.

Juberthie C. \& Decu V. (Eds.), 1994, 1998, 2001. Encyclopaedia Biospeologica
$1: 1-834 ; 2: 835-1374 ; 3: 1375-$ 2294; Soc. Intern. Biospeol., Bucarest (Roumanie) et Moulis (France) : $2294 \mathrm{pp}$.

Karaman S.G. \& Pesce G.L., 1980. Researches in Africa by the Zoological Institute of l'Aquila, Italy, V- On three subterranean Amphipods from North Africa (Amphipoda, Gammaridae). Bull. Zool. Mus. Univ. Van Amsterdam 7 (20) : 197-207.

Koenemann S. \& Holsinger J.R., 2001. Systematics of the North American subterranean amphipod genus Bactrurus (Crangonyctidae). Beaufortia (Bull. Zool. Mus. Univ. Amsterdam) 51 (1) : 1-56.

Magniez G., 1979. Metastenasellus powelli sp.n. A new stenasellid Isopod Crustacean from littoral groundwaters of Southern Nigeria. Crustaceana 37 (3) : 265-276.

Maguire B. Jr., 1964. Crustacea : a primitive Mediterranean group also occurs in North America. Science 146 : 931-932.

Messouli M., 1984. Recherches sur la faune aquatique endogée des sources du Haouz. Mém. C.E.A. Fac. Sc. Semlalia. Marrakech, $46 \mathrm{pp}$.

Messouli M., 1988. Les Crustacés Amphipodes souterrains du Groupe Metacrangonyx. Répartition, Systématique, Phylogénie. Thèse de $3^{\mathrm{e}}$ Cycle, Fac. Sc. Marrakech, $220 \mathrm{pp}$.

Messouli M., 1994. Évolution, Phylogénie et Biogéographie historique des Metacrangonyctidae, Crustacés Amphipodes stygobies du Nord de l'Afrique et des régions voisines. Thèse de doctorat d'État, Fac. Sc. Marrakech, $311 \mathrm{pp}$.

Monod Th., 1924. Sur un type nouveau de Malacostrace, Thermosbaena mirabilis nov. gen., n. sp. Bull.Soc. Zool. France 49 : 58-68.

Monod Th., 1927. Thermosbaena mirabilis Monod. Remarques sur sa morphologie et sa position systématique. Faune des Col. Françaises 1 : 29-51. 
Monod Th., 1930. Contribution à l'étude des "Cirolanidae". Ann. Sc. Nat. Zool. 10 (13) : 129-183.

Nourrisson M., 1956. Etude morphologique comparative et critique des Typhlocirolana (Crustacés Isopodes Cirolanides) du Maroc et de l'Algérie. Bull. Soc. Sci. Nat. Phys. Maroc. 36 : 103-124.

Pesce GL., Tete P. \& De Simone M., 1981. Ricerche in Africa dell' Istitut di Zoologia de l'Aquila. VI. Ricerche, faunistische in acque sotterranee del Magreb (Tunisia, Algeria, Morroco) e, dell Egitto. Natura. Soc. Ital. Sci. Nat., Museo. Civ. Stor. Nat. E Acquar. Civ. Milano. 72 (1-2) : 63-69.

Racovitza E.G., 1912. Cirolanides (1ère série). Arch. Zool. Exp. et Gen. 10 (5) : 203-205.

Rodier J., 1984. L'analyse de l'eau, eaux naturelles, eaux résiduaires et eau de mer. $7^{e}$ Éd. Dunod, Paris, 1365 pp.
SPSS, 1994. SPSS 10.0.5, SPSS 6.1 for Windows Update. SPSS Inc. Chigago.

Steube Ch., Richter S. \& Grieber Ch., 2009. First attempts towards an integrative concept for the ecological assessment of groundwater ecosystems. Hydrogeology Journal 17 : 23-35.

Sullivan P.J., 1982. Dilution of municipal landfill leachate in Franklin County. Indiana. J. of Environ. Health. V (44) : 253-257.

Vandel A., 1964. Biospéologie : la biologie des animaux cavernicoles. Gauthier Villars, Paris, 620 pp.

Wilkens H., Culver D.C. \& Humphreys W.F. (Eds.), 2000. Ecosystems of the World 30 : Subterranean ecosystems. Elsevier, New York, 791 pp.

Zébazé-Togouet S.H., Boutin Cl., Njiné T., Kemka K., Nola M. \& Foto Menbohan S., 2009. First data on the groundwater quality and aquatic fauna of some wells and springs from Yaoundé (Cameroun). Eur. Journ. Water Quality 40 : 51-74. 